\title{
Advanced Fresnel X-ray telescopes for spectroscopic imaging
}

\author{
Christoph Braig • Peter Predehl
}

Received: 27 March 2008 / Accepted: 20 October 2009 / Published online: 19 November 2009

(C) The Author(s) 2009. This article is published with open access at Springerlink.com

\begin{abstract}
We present an effective achromatic imaging technique for diffraction-limited X-ray telescopes. For a common focal length, independent optical sections of the aperture are dedicated to two or more spectral bands and optimized with respect to their efficiency. For this purpose, we introduce the "achromatic gain" of an X-ray hybrid lens. Large-scale segmented and nested apertures provide a promising implementation of that scheme. An optimized numerical example and astrophysical simulations prove its capabilities for an energy range between 6 and $16 \mathrm{keV}$.
\end{abstract}

Keywords Fresnel lenses • X-ray optics • Instrumentation • High resolution imaging techniques

\section{Introduction}

Fresnel lenses and their derivatives have been suggested for next generation $\mathrm{X}$-ray telescopes $[1,2]$. Being inexpensive and lightweight, diffraction limited optics with an angular resolution down to $10^{-3}$ or even $10^{-6}$ arcsec may be manufactured from low-Z lens materials like beryllium $(\mathrm{Be})$ or even ordinary plastics. Regarding Abbe's law, aperture diameters of a few decimeters would

C. Braig $(\bowtie)$

Friedrich-Schiller-Universität, 07745 Jena, Germany

e-mail: christoph.braig@uni-jena.de

P. Predehl

Max-Planck-Institut für Extraterrestrische Physik, 85748 Garching, Germany 
Table 1 Total transmission $\mathcal{T}_{1}(s)$ of achromatic hybrid lenses for $1 \leq s \leq 7$

\begin{tabular}{llllllll}
\hline $\mathrm{s}$ & 1 & 2 & 3 & 4 & 5 & 6 & 7 \\
$\mathcal{T}_{1}(s)$ & $78.7 \%$ & $63.2 \%$ & $51.8 \%$ & $43.2 \%$ & $36.7 \%$ & $31.7 \%$ & $27.7 \%$ \\
\hline
\end{tabular}

The absorption of the diffractive component is neglected

be sufficient beyond photon energies of about $1 \mathrm{keV}$. Preliminary calculations $[1,3]$ were followed up by novel schemes [4] for hard-X and Gamma-ray applications. Recent papers analyzed specific aspects [5] and proposed an optimized design for simple transmissive X-ray telescopes [6].

However, little success has been made in the improvement of the dispersion limited spectral bandwidth. Even the bandwith of achromatic doublets is restricted to few $10^{2} \mathrm{eV}$ below $20 \mathrm{keV}$. For scientific purposes, multi-band telescopes that cover two or more energy intervals simultaneously are of great interest.

In this paper a possible improvement is discussed. We review fundamentals of diff ractive-refractive X-ray lenses and outline principles of segmented largescale apertures. Such optics provide all conditions for the development of nested two-band achromatic telescopes. We introduce the "achromatic gain" as the central concept of such common-focus instruments. An optimized configuration is designed, briefly analyzed with respect to material insufficiencies and applied to an astrophysical target. The simulation, performed on the central region of a galaxy, demonstrates the usability of our scheme.

Due to the large quantity of symbols within this work, we overview the most important definitions in Table 1 at the end of Section 2.

\section{Hybrid Fresnel lenses}

Diffractive lenses may be regarded as circular diffraction gratings. For an aperture radius $R$ and a photon energy $E=h c / \lambda$, the focal length $F_{Z}$ is given as:

$$
F_{Z}=R^{2} N^{-1} E(h c)^{-1},
$$

where $h$ and $c$ denote Planck's constant and the vacuum velocity of light. The geometrical zone number $N \gg 1$ counts the number of phase shifts $\Delta \varphi=\pi$ under which the aperture is seen from the focal point. Binary zone plates (ZP) [7] and even phase ZPs [8] distribute main fractions of the incoming flux to diffraction orders different from the first. Only $10 \%$ in case of binary ZPs or $41 \%$ for phase ZPs can be used for imaging. Fresnel lenses, i.e. blazed circular diffraction gratings, provide an efficiency up to $100 \%$, with the exception of absorption losses. 


\subsection{Fresnel diffraction in the X-ray regime}

The field amplitude $\mathcal{A}(E)$ on the optical axis in a distance $z$ from the Fresnel lens plane can be obtained from the incoming amplitude, which is transformed by the Fresnel lens to $\mathcal{A}_{0}(r)$ and directed to the focal plane [9]:

$$
\mathcal{A}(E)=i \frac{k}{z} \int_{0}^{R} \mathcal{A}_{0}(r) \exp \left(-i \frac{k}{2 z} r^{2}\right) r d r .
$$

In (2), the wave number is given as $k \equiv 2 \pi / \lambda$ and the transformed amplitude can be written as $\mathcal{A}_{0}(r)=\exp \left(-i k(n-1) t_{Z}(r)\right)$ for an index of refraction $n=1-\delta-i \beta$. We neglect absorption losses for pure diffractive lenses, i.e. $\beta=0$. The stepwise Fresnel profile function for a blaze energy $E_{c}$ reduces the lens thickness by integer multiples of the $2 \pi$-thickness every 2 nd Fresnel zone:

$$
t_{Z}(r)=\frac{r^{2}}{2 F_{Z} \delta\left(E_{c}\right)} \bmod t_{2 \pi} \quad, \quad t_{2 \pi} \equiv \frac{h c}{E_{c} \delta\left(E_{c}\right)} .
$$

The real contribution $\delta$ to the refractive index follows a normal dispersion $\propto$ $E^{-2}$. Generalized to normalized energies $\psi \equiv E / E_{c}$, the numerical value $\mathcal{A}(\psi)$ of the axial field amplitude is obtained from the phase shift across all Fresnel rings. The radius $r$ is transformed via $r \equiv \tau R$ with $0 \leq \tau \leq 1$ :

$$
\mathcal{A}(\psi)=\mathcal{C} \sum_{n=1}^{N / 2} \exp \left(2 \pi i \frac{1-n}{\psi}\right) \int_{\tau_{n}}^{\tau_{n+1}} \mathcal{F}_{0}(\psi, \tau) \tau d \tau .
$$

The function $\mathcal{F}_{0}(\psi, \tau) \equiv \exp \left(-i \pi N\left(m-\psi^{-1}\right) \tau^{2}\right)$ contains the diffraction order $m$, which is introduced via

$$
F_{Z}^{(m)}(\psi)=m^{-1} \psi F_{Z}\left(E_{c}\right),
$$

according to (1). The proportionality constant in (4) is defined as $\mathcal{C} \equiv 2 \pi i m N$. Since one Fresnel ring contains two (binary) Fresnel zones, the phase shift must be integrated for $\tau_{n}=\sqrt{2(n-1) / N}$, with $1 \leq n \leq N / 2$. The total amplitude would be given as the coherent superposition from the contributions of all individual Fresnel rings. Wherever the condition $m=\psi^{-1}$ is fulfilled, incoming X-rays are regularly focused to an image point $z$ on the optical axis at a normalized distance $\zeta \equiv z / F$, where $\zeta>0$ and $F=F_{Z}^{(1)}(1)$ denotes the focus for $m=1$ and an energy $\psi=1$ (see Fig. 1). From (4), we obtain the efficiency $P_{m}(\psi)$,

$$
P_{m}(\psi)=\left|\frac{\mathcal{A}(\psi)}{\pi m N}\right|^{2} \quad \text { with } \quad \sum_{m=-\infty}^{\infty} P_{m}(\psi)=1,
$$


Fig. 1 The phase condition for blazed Fresnel X-ray lenses with normal dispersion $\delta \propto E^{-2}$. The deflected wave fronts are shown for one Fresnel ring and the first three diffraction orders (exaggerated drawing)
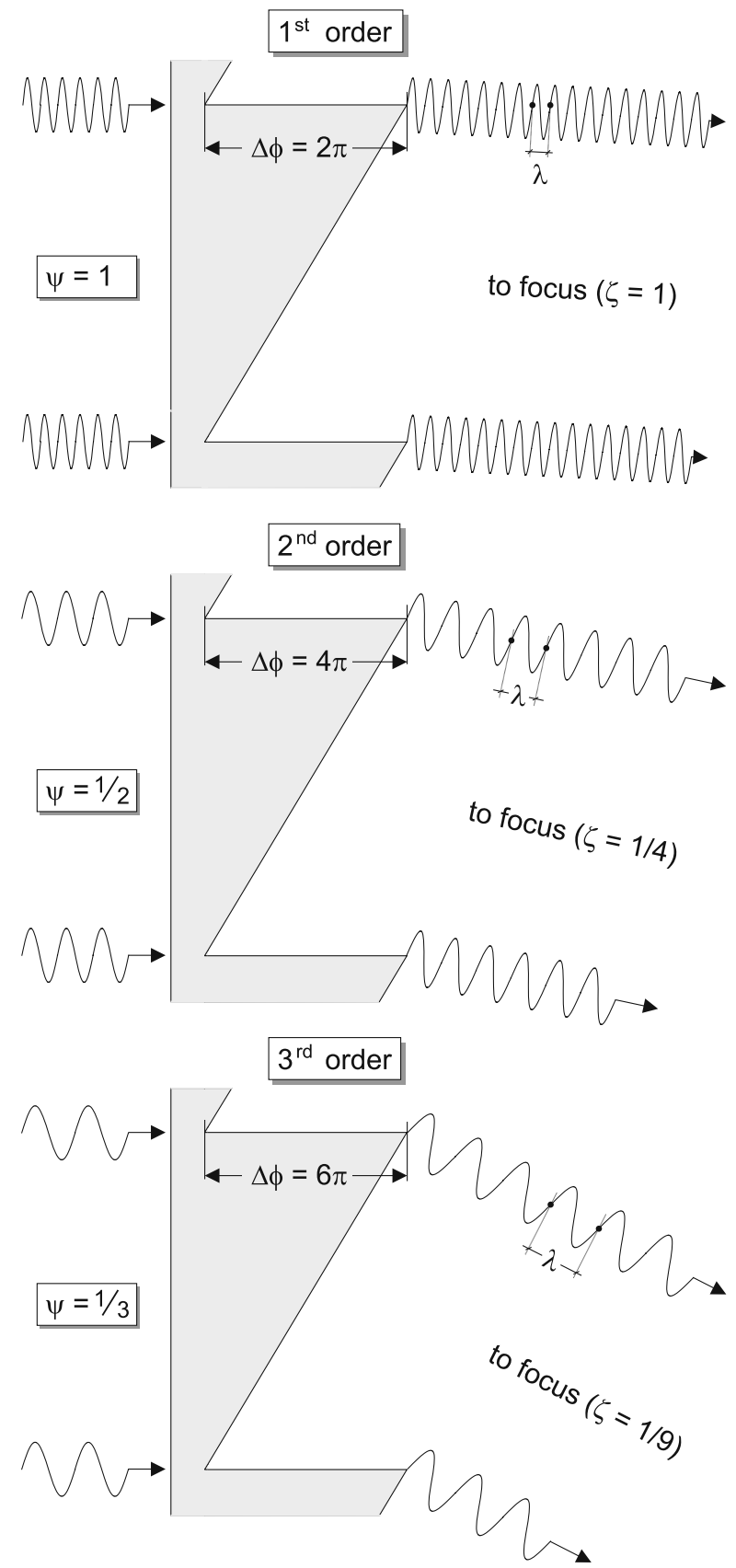

since the point spread function (PSF) in the plane $\zeta$ always shows an Airy shape whose peak intensity scales with $(\pi m N)^{2}$ whereas the spot size $\varnothing_{P S F}$ decreases with $(\pi m N)^{-1}$. So we get the fraction $P_{m}(\psi)$ of the incoming flux 
Fig. 2 Focusing efficiency $P_{m}(\psi)$ of diffractive X-ray lenses without absorption. For blazed Fresnel lenses the first three diffraction orders are shown according to (7)

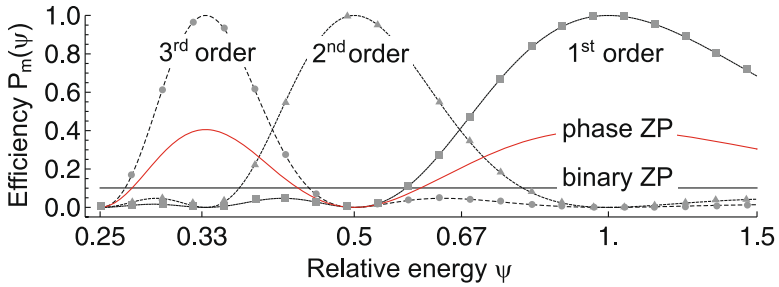

which is found in the diffraction order $m$ from (6). For all diffraction orders $m \geq 1$, this focusing efficiency $P_{m}(\psi)$ follows the "sinc" function [5]:

$$
P_{m}(\psi)=\left(\frac{\sin \left(\pi \mathcal{H}_{m}(\psi)\right)}{\pi \mathcal{H}_{m}(\psi)}\right)^{2}, \mathcal{H}_{m}(\psi) \equiv m-\frac{1}{\psi} .
$$

Aside from integer fractions with $\psi=m^{-1}$, the blaze condition is not fulfilled for the Fresnel grooves, and the X-rays are distributed to several diffraction orders, as shown in Fig. 2.

\subsection{Achromatic hybrid optics}

Dispersion corrected achromatic X-ray systems are made of closely spaced diffractive $(Z)$ and divergent refractive $(L)$ devices whose focal lengths are related as $F_{L}=-2 F_{Z}$ [2]. This setup is sketched in Fig. 3. Within the thin lens appproximation, the focal lengths can be added via $F^{-1}=F_{Z}^{-1}+F_{L}^{-1}$ and we obtain:

$$
F_{m}(\psi)=2 F_{Z}\left(2 m \psi^{-1}-\psi^{-2}\right)^{-1}
$$

for the diffraction order $m$. In Fig. 4, this function is compared to the linear dispersion of a pure diffractive Fresnel lens. Within the diffraction limited focal depth of field (DOF) [5,7], the spectral width is enlarged from $\Delta E=E / N$ for pure diffractive optics to $\Delta E=2 N^{-1 / 2} E$ for achromatic lenses. Since massive lens components cause absorption-absorption losses of the very thin diffractive Fresnel lens component are neglected within this work-the geometrical zone number $N$ of the (diffractive) lens should be compared to an intrinsic parameter of the material, the "critical zone number" $N_{0}$. As defined

Fig. 3 Achromatic hybrid $\mathrm{X}$-ray lens, as suggested by Skinner [2] and Wang et al. [10]. An additional support structure will be neglected within this work. The focal plane of our telescope contains several $10^{2}$ resolution elements in diameter

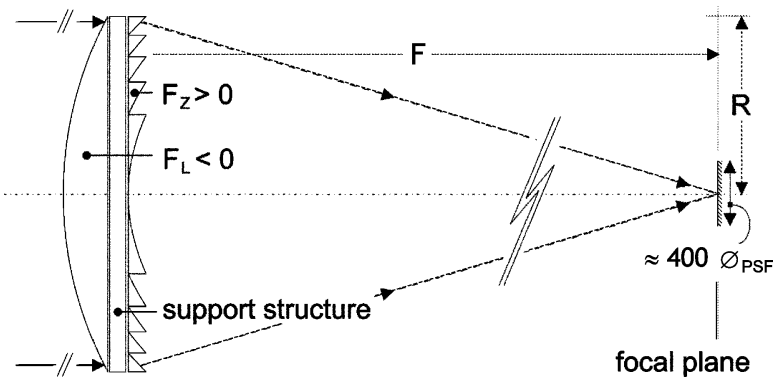


Fig. 4 Diffractive

(dotted) and achromatic (solid) normalized focal length dispersion $\zeta$ as a function of $\psi$ for the first three diffraction orders. For blazed Fresnel lenses, the $\mathrm{X}$-ray light is completely concentrated to only one of these orders $1 \leq m \leq 3$ wherever $\mathcal{H}_{m}(\bar{\psi})=\overline{0}$

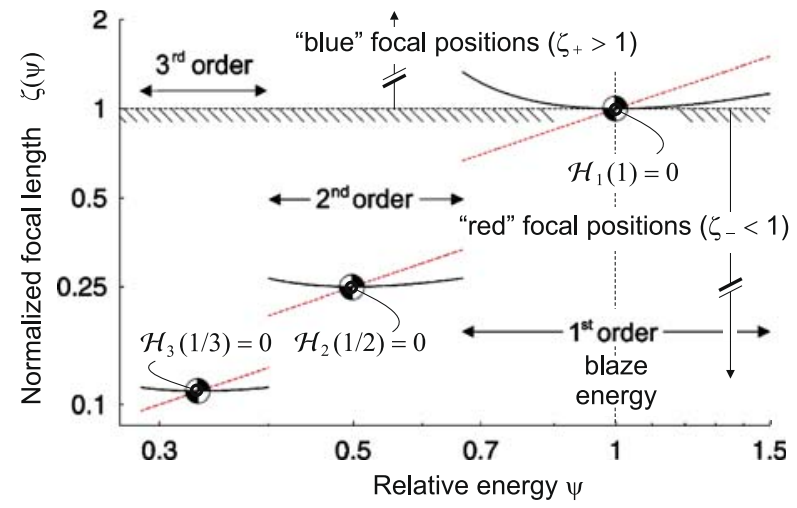

by Yang [9], we set $N_{0} \equiv \delta /(2 \pi \beta)$, for an imaginary part $\beta$ of the refractive index $n$. The total photon transmission may be calculated by integration of the punctual transmission over the lens radius,

$$
\mathcal{T}(s)=\frac{2}{R^{2}} \int_{0}^{R} \exp \left(-\frac{4 \pi}{\lambda} \beta t_{L}(r)\right) r d r .
$$

In (9), the radial profile of the convex spherical correction lens is given as $t_{L}(r)=\left(R^{2}-r^{2}\right) /(2 \xi)$, where we used the lens curvature $\xi \equiv F \delta\left(E_{c}\right)$. As for the diffractive (Fresnel) component we assume an ordinary dispersion $\delta(E) \propto E^{-2}$ for the refractive device, too. With the definition of $s \equiv N / N_{0}$, (9) yields

$$
\mathcal{T}(s)=2 \int_{0}^{1} \exp \left(-\frac{1}{2} \psi^{-1} s\left(1-\tau^{2}\right)\right) \tau d \tau .
$$

Since the achromatic hybrid lens produces properly focused images only for energies $\psi^{-1}=m$, the result may be expressed in parametrical dependence on the diffraction order $m$. Apart from that, the total transmission is an exclusive function of the zone ratio $s$ :

$$
\mathcal{T}_{m}(s)=2 m^{-1} s^{-1}(1-\exp (-m s / 2)) .
$$

The product $m s \propto m N$ can be interpreted with respect to the Fresnel lens: In case of higher orders with $m \geq 1$, the zone number $N$ must be replaced by $m N$, which counts the effective number of phase shifts $\Delta \phi=\pi$ over the lens radius (see Fig. 1). Table 2 provides data on the hybrid lens transmission for the first diffraction order.

\section{Segmented apertures}

In case of large apertures with diameters beyond $\sim 2 \mathrm{~m}$, it is useful to replace common compact lenses by segmented optics. In the following, we review and extend the detailed considerations of $[5,6]$. 
Table 2 Global symbols and definitions used within this work

Their usage is given for most common applications. The notation $[\ldots]$ describes the dimension unit of a symbol

\begin{tabular}{lll}
\hline Symbol & Description & Usage \\
\hline$R$ & Outer lens radius & \\
$r_{\text {obs }}$ & Radius of central obstruction & $r_{\text {obs }} \leq R$ \\
$a$ & Fractional lens obstruction & $a \equiv r_{\text {obs }} / R$ \\
$T_{N}$ & \#(segment rings) & \\
$k$ & Index of segment rings & $1 \leq k \leq T_{N}$ \\
$\eta_{N}$ & Segmented conversion & $\eta_{N}(a) \lesssim 3$ \\
$E_{c}$ & Fresnel blaze energy & \\
$\psi$ & Relative energy & $\psi \equiv E / E_{c}$ \\
$\Delta E$ & Absolute spectral width & {$[\Delta E]=\mathrm{eV}$} \\
$F$ & Focal length at energy $E_{c}$ & \\
$\zeta$ & Fractional focal distance & $\zeta \equiv z / F$ \\
$m$ & Diffraction order & $m \geq 0$ \\
$N$ & \#(geometrical Fresnel zones) & $R^{2}=N \lambda F$ \\
$N_{\star}$ & \#(Fresnel zones) per segment & $N=T_{N} N_{\star}$ \\
$N_{0}$ & Critical zone number & $N_{0}=\delta /(2 \pi \beta)$ \\
$s$ & Zone ratio & $s \equiv N_{(\star)} / N_{0}$ \\
$E_{0}$ & Basic multi-band energy & \\
$\psi_{0}$ & Relative multi-band energy & $\psi_{0} \equiv E / E_{0}$ \\
$E_{p, q}$ & Multi-band blaze energies & $E_{p, q} \propto E_{0}$ \\
$r_{\text {lens }}^{(i, o)}$ & Inner, outer partial lens radius & $r_{\text {lens }}^{(i)}<r_{\text {lens }}^{(o)}$ \\
$\varnothing_{\mathrm{FOV}}$ & Detector diameter & $\varnothing_{\mathrm{FOV}}=2 r_{\mathrm{FOV}}$ \\
$n_{\mathrm{FOV}}$ & \#(resol. elements) in FOV & $n_{\mathrm{FOV}} \propto \varnothing_{\mathrm{FOV}}$ \\
$\varnothing_{\mathrm{PSF}}$ & Focal spot size (HEW) & {$\left[\varnothing_{\mathrm{PSF}}\right]=\mathrm{mm}$} \\
$\Delta \epsilon$ & Angular resolution & $\Delta \epsilon=\varnothing_{\mathrm{PSF}} / F$ \\
$w$ & \#(coh. steps) within segment & \\
$\mathcal{T}$ & Mean lens transmission & $\mathcal{T}=\mathcal{T}_{(w)}(s)$ \\
$\mathcal{G}$ & Achromatic gain & $\mathcal{G}=\mathcal{G}\left(N, N_{0}\right)$ \\
$A_{\text {eff }}$ & Effective lens area & $A_{\mathrm{eff}}=\pi R^{2} \mathcal{T}(s)$ \\
\hline & &
\end{tabular}

\subsection{Conventional segmentation}

Convenient segmented apertures are divided into $T_{N}$ rings of constant zone numbers $N_{\star}=N / T_{N}$, where $N$ denotes the total Fresnel zone number. Each of those rings, counted by $1 \leq k \leq T_{N}$, contains an increasing number of segments, so that their shapes approximate squares for sufficiently large $k \gg 1$ (see upper left image in Fig. 5). In order to minimize absorption losses, the radial thickness profile of an ordinary, convex refractive lens component should be reduced periodically with respect to subsequent segment rings. The resulting radial cross section of such conventional segmented apertures is sketched below the lens shape in Fig. 5. Our suggested "sandwich design" contains the diffractive lens within two symmetric refractive lens components. The maximum thickness of the lens is reduced as follows:

$$
\Delta t_{\max }=\frac{N_{\star}}{4} \frac{h c}{E_{c} \delta\left(E_{c}\right)},
$$

and the overall transmission is still given by (11) for the modified parameter $s \equiv N_{\star} / N_{0}$. 
Fig. 5 Geometrical structure and spectral properties of an obstructed, segmented aperture for conventional (left) and coherently stepped versions (right). This example assumes $T_{N}=7$ segment rings, from which $k_{o b s}=3$ are obstructed. The coherently stepped design is shown for $w=3$ steps within one segment. Axial symmetry is presumed, $0 \leq \varphi<2 \pi$
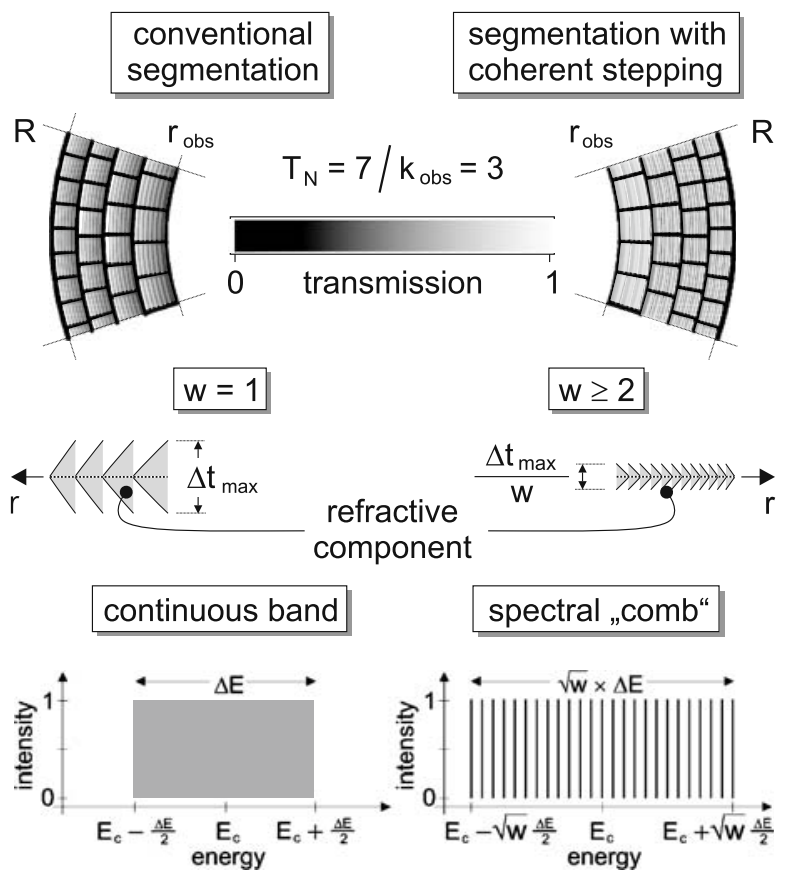

As it was calculated elsewhere [6], the detectable spectral bandwidth within the DOF is given as

$$
\Delta E=2 N_{\star}^{-1 / 2} E,
$$

in analogy to compact achromatic hybrid lenses. The spectral band from (13) is also illustrated on the left in Fig. 5. Within this energy range, the PSF of the segmented lens may be recorded with an accuracy near the diffractionlimit which is constrained by the segment size and the non-uniform gray-wedge transmission due to the refractive lens profile.

\subsection{Segmentation with coherent stepping}

For some instrumental applications, e.g. large apertures or short focal lengths, large zone numbers $N$ are desirable whereas reasonable segment sizes should still ensure a sufficient spatial and angular resolution. As a consequence, high $s$-ratios, again defined as $N_{\star} / N_{0}$, with radial transmission gradients close to $100 \%$ within individual segments are required. Wherever such technical constraints cause significant absorption losses for $s \gg 1$, an additional "coherent stepping" of the refractive component within each segment may conserve the focal spot size and an acceptable transmission as well. This advanced scheme is compared to the conventional case on the right in Fig. 5. We denote the number of steps or "teeth" within one segment by an integer $w$. Each of these 
coherent steps contains $N_{\star} / w$ Fresnel zones and the maximum thickness is reduced to:

$$
\frac{\Delta t_{\mathrm{max}}}{w}=\frac{N_{\star}}{4 w} \frac{h c}{E_{c} \delta\left(E_{c}\right)},
$$

with respect to the conventional segmentation scheme. In extension of (11), the total transmission should be written as:

$$
\mathcal{T}_{m}^{(w)}(s)=2 m^{-1} w s^{-1}\left(1-\exp \left(-m w^{-1} s / 2\right)\right)
$$

In other words, the $s$-ratio $N_{\star} / N_{0}$ is reduced by the factor $w$ to an "effective" value $s / w$.

Since all refractive steps within one segment now act coherently, interference effects lead to an oscillating spectral response in the focal plane [2]. The diffraction-limited PSF is only found for certain energies:

$$
\psi_{n}=1 \pm 4 n \frac{w}{N_{\star}} \quad \text { with } \quad n \geq 0
$$

where $w \geq 2$ determines the number of spectral spikes and their constant spacing $4 w / N_{\star}$. The resulting comb of energies spreads over a total bandwidth which is enlarged by a factor of $\sqrt{w}$ with respect to the conventional version $(w=1)$. The net bandwidth which effectively contributes to the luminous power is therefore given as:

$$
\Delta E=\sqrt{w} \frac{1}{2 w} \frac{2 E}{\sqrt{N_{\star}}}=\frac{E}{\sqrt{w N_{\star}}} .
$$

An example is shown on the bottom in Fig. 5. Such comb structures may be properly detected with a spectral resolution $\Delta E_{\text {comb }}=2 E / N_{\star}$. Special detection techniques beyond conventional CCDs are required for that purpose. Promising candidates include transition edge sensors (TES) and superconducting tunnel junction (STJ) devices and will be discussed elsewhere (Braig and Predehl, unpublished manuscript).

\subsection{Angular resolution of segmented lenses}

The spatial and angular resolution provided by an incoherently assembled aperture-with or without coherent stepping-is governed by the "mean"

Table 3 Conversion for segmented apertures

\begin{tabular}{llllllll}
\hline $\mathrm{a}$ & 0 & 0.1 & 0.2 & 0.4 & 0.6 & 0.8 & 1.0 \\
$\eta_{N}$ & 2.27 & 2.30 & 2.35 & 2.55 & 2.86 & 3.19 & 3.55 \\
\hline
\end{tabular}

The geometrical correction $\eta_{N}$ depends on the central obstruction a 
Fig. 6 Fourier transformation (right) of almost squared shaped and rotated segments (left). That example assumes $s=30, w=3$

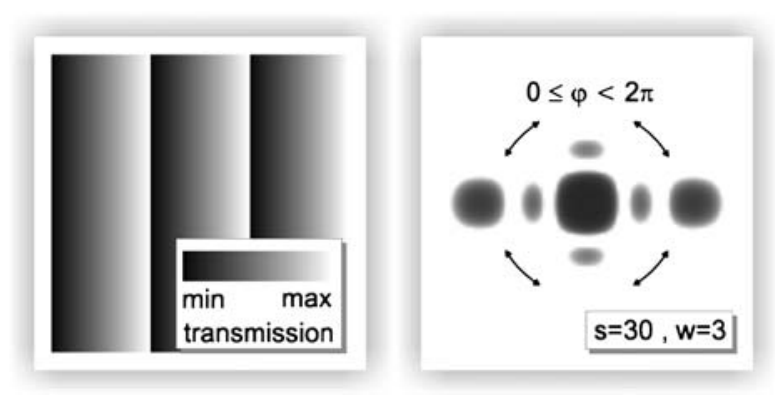

segment size, in contrast to common lenses, whose diffraction-limited angular resolution scales with the reciprocal lens radius $R^{-1}$ : Since all single segments act independently, an optical coherence is exclusively maintained therein.

The actual angular resolution depends not only on the-slightly varyingsize of the lens segments but also on the radial transmission gradient therein. The contribution of such an achromatic prism-like device to the focal point spread function coincides with the squared Fourier transform of its amplitude transmission. The latter depends on the radial coordinate $r$ within the lens according to Fig. 5. As it was shown in detail elsewhere [5, 6], the diffraction pattern in the focal plane can be approximated assuming almost squared shaped segments for ring numbers $k \gtrsim 3$, averaged along $0 \leq \varphi<2 \pi$.

In summary, the focal spot of conventional as well as coherently stepped segmented apertures is a function of the effective geometrical segment size and the non-uniform absorption due to the refractive lens profile. Since both features affect the PSF independently, their contributions can be factorized.

In a first approach, we neglect any absorption and restrict to the geometrical contribution caused by segmentation. Previous investigations [5] proved a linearity of the incoherent angular resolution $\Delta \epsilon_{\text {inc }}$ in the ring number $T_{N}$, modified by an empirical correction $\eta_{N}(a)$. This parameter stands for radial decreasing segment sizes and obstructions $0 \leq a \leq 1$, as listed in Table 3. With respect to the well known Abbe limit $\Delta \varepsilon_{\text {coh }}$ of compact apertures with the same radius, we obtain

$$
\Delta \epsilon_{\mathrm{inc}} \approx \eta_{N}(a) T_{N} \Delta \epsilon_{\mathrm{coh}},
$$

where $\Delta \epsilon_{\mathrm{coh}}=\alpha \lambda R^{-1}$ describes the angular resolution of the coherent analogue. The HEW is associated with $\alpha=0.535$.

Table 4 Relative angular resolution for segmented hybrid optics without reduction $(w=1)$ for various $s$-ratios

\begin{tabular}{llllllllll}
\hline$s$ & 1 & 2 & 3 & 4 & 5 & 6 & 7 & 8 & 9 \\
$Q_{1}$ & 1.00 & 1.01 & 1.03 & 1.06 & 1.09 & 1.12 & 1.17 & 1.21 & 1.27 \\
\hline
\end{tabular}


Table 5 Relative angular resolution for coherently stepped segments, based on absorption

\begin{tabular}{|c|c|c|c|c|c|c|c|c|c|c|}
\hline 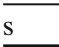 & 5 & 10 & 15 & 20 & 25 & 30 & 35 & 40 & 45 & 50 \\
\hline$Q_{5}$ & 1.00 & 1.02 & 1.04 & 1.06 & 1.10 & 1.15 & 1.21 & 1.28 & 1.36 & 1.48 \\
\hline$Q_{10}$ & 1.00 & 1.00 & 1.01 & 1.02 & 1.03 & 1.04 & 1.05 & 1.07 & 1.08 & 1.10 \\
\hline
\end{tabular}

Deviations $Q_{w}(s)$ less than $2 \times 10^{-2}$ are neglected

Next we take the absorption of the refractive lens component into account. In general, the HEW will suffer from the non-uniform, i.e. periodical graywedge transmission. The relative spatial resolution $Q_{w}(s)$ of an arbitrary, squared-shaped segment should be calculated using a two-dimensional Fourier transformation of the amplitude transmission function [6]. The diffraction pattern is obtained from rotated and superposed segments, as shown in Fig. 6. The factor $Q_{w}(s)$ describes how much the focal spot size (HEW) is enlarged by absorption with respect to a fully transparent segment of the same size and must be calculated numerically for all relevant $s$-ratios and $w$-numbers. Some data are listed in Table 4 for segments without reduction $(w=1)$ and Table 5 for coherently stepped versions $(w \geq 5)$. Obviously, coherent stepping becomes useful in particular for s-ratios beyond $\sim 10$; strong reductions $w \gtrsim 5$ conserve the resolution of the ideal transparent segment to a far extent. Using $Q_{w}(s)$, the focal spot size of arbitrary segmented hybrid lenses can be estimated. Finally, we write the resulting equation in the form:

$$
\Delta \epsilon_{\mathrm{inc}} \approx \eta_{N}(a) T_{N} Q_{w}(s) \Delta \epsilon_{\mathrm{coh}}
$$

Compared to an extremely time-consuming accurate numerical calculation, which means the direct Fourier transform of the complete segmented aperture, the factorized approximation from (19) works well within an error range of $10^{-2}$.

It should be noted that "segmentation" does not necessarily mean an incoherent superposition of the focal spots provided by all lens segments-if accurately aligned, the phase condition would be preserved over the whole aperture and the Abbe limit $\Delta \varepsilon_{\text {coh }}$ is still valid. However, this fine adjustment on a scale of microns will cause substantial problems in space and is therefore considered to be practically impossible.

\section{Dual band apertures}

Many astronomical investigations benefit from multi-wavelength observations. For instance, insights into conversion mechanisms of AGN or X-ray binaries may arise from hardness ratios. Simple achromatic two-band telescopes for such applications use the common-focus concept mentioned above. Its theory is based on the one developed for purely diffractive apertures [5]. We will repeat the main results in short. 
A Fresnel lens blazed on an energy $E_{p}$ focuses mistuned X-rays with an energy $E_{q} \neq E_{p}$ to other, often numerous, diffraction orders, according to (7). The fractional achromatic focal length dispersion for $\zeta_{ \pm} \equiv z / F$, where $z$ denotes the focus of the scattered light,

$$
\zeta_{ \pm}=\left(2 m \psi^{-1}-\psi^{-2}\right)^{-1},
$$

distinguishes "red" $\left(\zeta_{-}<1\right)$ from "blue" $\left(\zeta_{+}>1\right)$ focal positions, as it follows from (8). The geometry is shown in Fig. 7. In the focal plane, those defocused $\mathrm{X}$-rays evoke ring-like halos around the detector. In order to protect the active detector field from any scattered X-rays, the aperture must be divided into partial ring lenses, each of them dedicated to one of the desired energy bands. We consider an obstructed lens with an outer radius $r_{\text {lens }}^{(o)}$ :

$$
r_{\text {lens }}^{(i)}=a r_{\text {lens }}^{(o)}, \quad \text { with } \quad 0 \leq a \leq 1
$$

defines the inner radius of that lens partition. Simple geometric formula, in particular the theorem on intersecting lines, prove the condition:

$$
a r_{\text {lens }}^{(o)} \geq \mathcal{S}\left(\zeta_{ \pm}\right) r_{\mathrm{FOV}} \quad, \quad 2 r_{\mathrm{FOV}}=n_{\mathrm{FOV}} \varnothing_{\mathrm{PSF}}
$$

for $n_{\mathrm{FOV}}$ resolution elements that may be detected across an "unsoiled" field of view, measured in units of the circular detector size $2 r_{\mathrm{FOV}}$. The scaling factor:

$$
\mathcal{S}\left(\zeta_{ \pm}\right) \equiv \frac{r_{ \pm}}{r_{\mathrm{FOV}}}= \pm 2\left(1-\zeta_{ \pm}^{-1}\right)^{-1}
$$

depends on the fractional focal length $\zeta \equiv z / F$, where the signs \pm are used as above. The lens radii $r_{ \pm}$correspond to $r_{\text {lens }}^{(i)}$ from (21).

Compared to simple diffractive Fresnel objectives, the stray light behavior of achromatic hybrid lenses is more complicated, as it follows from their focal length dispersion in (20). For a basic energy $E_{0}$, we obtain:

$$
\mathcal{S}_{m}\left(\psi_{0}\right)= \pm 2\left(1-\psi_{0}^{-1}\left(2 m-\psi_{0}^{-1}\right)\right)^{-1},
$$

with $\psi_{0} \equiv E / E_{0}$ and the diffraction order $m \in \mathbb{Z}$.

Fig. 7 Geometry and notation for detuned X-rays in dual-band telescopes. In general, different detector diameters are assumed for the soft and hard band, respectively

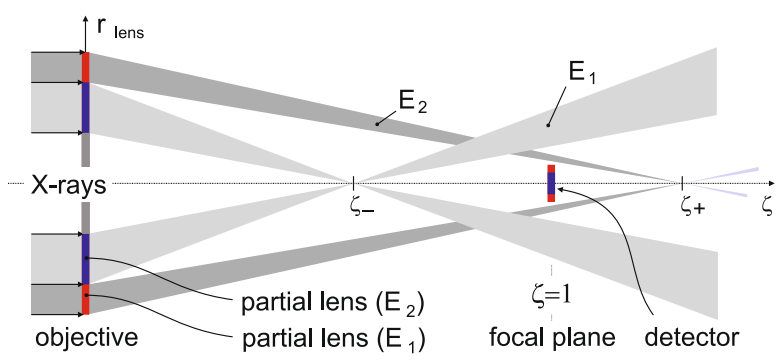




\section{The achromatic gain}

In Fig. 4 the normalized focal lengths of Fresnel lenses are shown for the first three diffraction orders.

\subsection{Definition for hybrid lenses}

However, this linear dispersion limits the diffractive spectral bandwidth $\Delta E=$ $E / N$, which is related to the DOF [5, 7]. Accordingly, the-photon limitedluminous power, defined as the product of the effective area and the spectral width:

$$
\left(A_{\mathrm{eff}} \times \Delta E\right)_{\mathrm{dif}}=P_{m}(\psi) \pi h c F_{Z}^{(m)}
$$

depends on the focal length $F_{Z}$.

The luminous power of an achromatic lens suffers from the serious absorption in the refractive lens device. In particular, we have:

$$
\left(A_{\mathrm{eff}} \times \Delta E\right)_{\mathrm{hyb}}=\pi R^{2} P_{m}(\psi) \mathcal{T}_{m}(s) \frac{2 E}{\sqrt{N_{(\star)}}},
$$

where the radius is given as $R^{2}=(N / 2) \lambda F$, since the achromatic focal length is twice as large as the diffractive one. Within this section, we use the index ( $\star$ ) for the zone number $N_{(\star)}$ wherever the formula is valid for compact and segmented hybrid lenses as well. Using (25) and (26), we define the achromatic gain $\mathcal{G}\left(N_{(\star)}, N_{0}\right)$ that relates the luminous power of the hybrid lens to the one of the solely diff ractive lens:

$$
\mathcal{G}\left(N_{(\star)}, N_{0}\right) \equiv \frac{\left(A_{\text {eff }} \times \Delta E\right)_{\text {hyb }}}{\left(A_{\text {eff }} \times \Delta E\right)_{\text {dif }}}=2 \sqrt{N_{(\star)}} \mathcal{T}_{m}(s) .
$$

In (27), we introduced the quantity $\mathcal{G}\left(N_{(\star)}, N_{0}\right)$ in order to compare the luminous power of two transmissive X-ray lenses which are based on the same diffractive component. An alternative definition would relate the luminous powers of achromatic hybrid lenses to their diffractive analogues with equal focal lengths. Though both options share advantages and drawbacks, we chose an experimental or technical approach: Existing, i.e. already produced Fresnel lenses can be easily corrected for chromatic focal length dispersion by additional refractive optics. In contrast, the detailed groove profile of real Fresnel lenses would always get the fingerprint of the difficult production process - and different Fresnel components would always be involved in case of an alternative definition based on equal focal lengths. 
Fig. 8 Achromatic gain for different critical zone numbers $400 \leq N_{0} \leq 1400$. The straight dotted line indicates the optimized arrangement with $N_{(\star)} \approx 2.5 N_{0}$

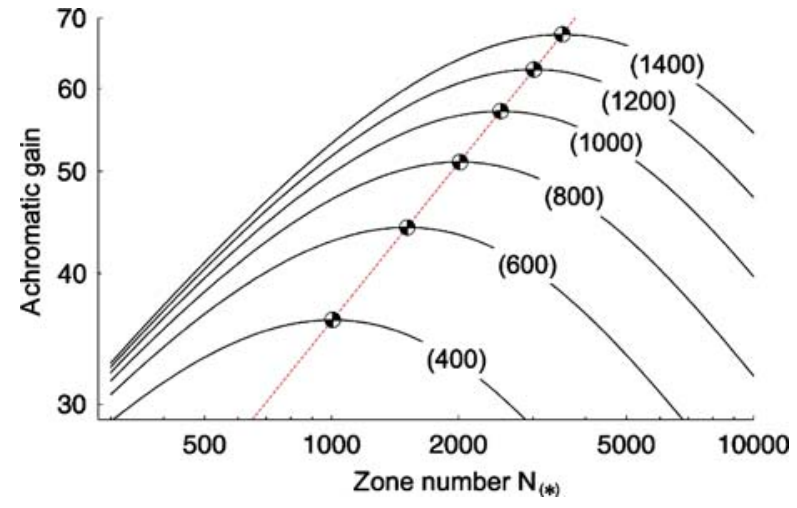

\subsection{Optimization of common-focus instruments}

The achromatic gain as it was introduced above is clearly independent of the Fresnel lens efficiency $P_{m}(\psi)$. We may use (27) and write the luminous power of the hybrid lens with $m=\psi^{-1}$ as:

$$
\left(A_{\mathrm{eff}} \times \Delta E\right)_{\mathrm{hyb}}=\pi \frac{h c}{2} \mathcal{G}\left(N_{(\star)}, N_{0}\right) F
$$

The subscript "hyb" will be neglected from now on. Interestingly, the luminous power depends on two quantities or "degrees of freedom" alone, namely $\mathcal{G}\left(N_{(\star)}, N_{0}\right)$ and the focal length $F$. The first one describes an internal feature of each individual hybrid lens. The second one is in common with other partial telescopes.

In (27) the spectral bandwidth and the absorption counteract each other as a function of $N_{(\star)}$, and an optimized achromatic gain is obtained for the condition $\partial_{N_{(\star)}} \mathcal{G}\left(N_{(\star)}, N_{0}\right)=0$. The result of the partial differentiation depends on $N_{0}$ :

$$
\mathcal{G}_{\text {opt }}\left(N_{(\star)}, N_{0}\right) \approx 1.8 \sqrt{N_{0}} \text { at } \quad N_{(\star)} \approx 2.5 N_{0} .
$$

In Fig. 8 the achromatic gains and its optimal values are illustrated for some typical values of $N_{0}$. Since the optical performance of low-Z materials like $\mathrm{Li}$ and Be peaks between 10 and $20 \mathrm{keV}$ with $N_{0} \sim 10^{3}$, the luminous power

Table 6 Zone numbers of $\mathrm{Li}$ and Be optics for a given focal length

\begin{tabular}{llllllllll}
\hline $\mathrm{E}(\mathrm{keV})$ & 4 & \multicolumn{1}{l}{6} & \multicolumn{1}{c}{8} & 10 & 12 & 14 & 16 & 18 & 20 \\
\hline$N_{\mathrm{opt}}^{(\mathrm{Li})}$ & 690 & 1582 & 2430 & 2898 & 3010 & 2888 & 2688 & 2480 & 2278 \\
$N_{\mathrm{opt}}^{(B e)}$ & 218 & 516 & 912 & 1326 & 1668 & 1884 & 1982 & 1994 & 1946 \\
\hline
\end{tabular}

The data optimize $A_{\text {eff }} \times \Delta E$ 
Fig. 9 Luminous intensity of achromatic X-ray lenses in the diffraction-limited operation mode for various gains $\mathcal{G}\left(N_{(\star)}, N_{0}\right)$. The line for $\mathcal{G}=16$ is a guide to the eye on the logarithmic scale. The lower limit with $\mathcal{G}_{\min }=4$ refers to an acceptable minimum (see text)

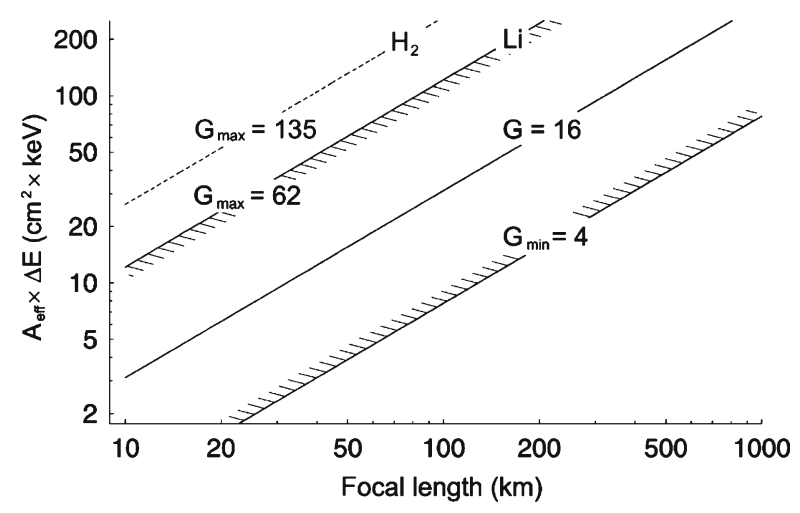

of an achromatic lens may be more than 50 times higher than for the solely diff ractive one. For a given energy, the material with the largest $N_{0}$ should be chosen. The following list overviews the maximum values $\mathcal{G}_{\max }$ for the lightest elements:

$$
\begin{array}{llll}
\mathrm{H}_{2} @ 2.90 & \mathrm{keV}: \mathcal{G}_{\max }=135 & \text { for } & N_{0}=5615 \\
\mathrm{He} @ 6.90 & \mathrm{keV}: \mathcal{G}_{\max }=84.0 & \text { for } & N_{0}=2164 \\
\mathrm{Li} @ 11.7 & \mathrm{keV}: \mathcal{G}_{\max }=62.5 & \text { for } & N_{0}=1199 \\
\mathrm{Be} @ 17.3 & \mathrm{keV}: \mathcal{G}_{\max }=50.9 & \text { for } & N_{0}=795
\end{array}
$$

In the soft X-ray range of a few $\mathrm{keV}$, liquid elements like $H_{2}$ and He provide the best performance but may hardly be exploited, at least in astronomical applications. Realistic X-ray optics might profit from solid-state materials like $\mathrm{Li}$ and $\mathrm{Be}$, at least if surface and bulk impurities are handled properly [6].

Within this work we assume crystals without impurities [6] and, as far as possible, zone numbers according to (29) as listed in Table 6. Telescopes with these optimized zone numbers would obtain maximum achromatic gain. Special applications might need minimized focal spots, however. A lower bound for the achromatic gain may be set around $\mathcal{G}=4$ because the corresponding Fresnel lens with the half focal length $F_{Z}=F / 2$ would reduce the focal spot size and the luminous power $A_{\text {eff }} \times \Delta E$ by the same factor, i.e. $50 \%$, according to (25). Thus one could also take the solely diffractive analogue instead of an achromatic one. Luminous intensities for various gains are shown in Fig. 9.

\section{An optimized design}

Based on (22), several possible solutions for the lens radii within the nested aperture can be found. In general, compared to purely diffractive systems [5], the obstruction for the outer lens is enlarged by $50-100 \%$ in case of an achromatic scaling factor. Following (24), the small ring lens for $E_{2}$ must be 
Fig. 10 Optimized obstruction of nested two-band telescopes. The obstructions for the two lowest diffraction orders ( $m=1$ and $m=2$ ) coincide for the condition $\mathcal{S}_{m_{ \pm}}\left(\psi_{0}\right)=2 \psi_{0}$ (straight dotted line $)$ at $\psi_{0} \approx 2.62$

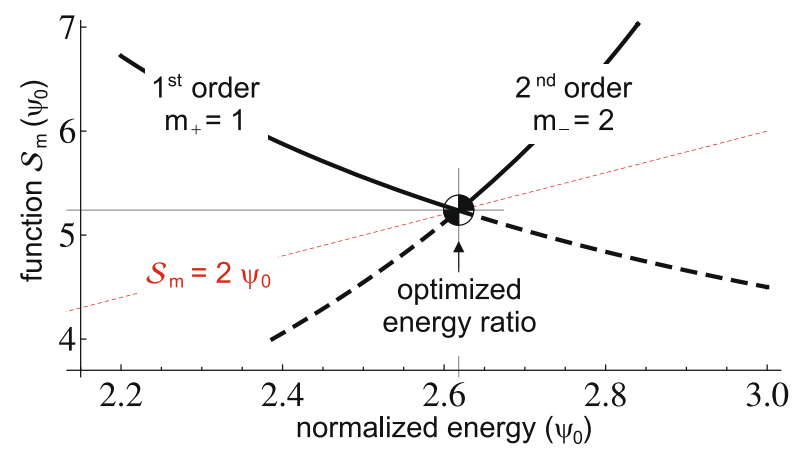

encircled by the large partial lens for the soft band at $E_{1}$. So it is desirable to find an optimized arrangement that favors the soft X-ray band.

\subsection{Lens parameters}

We ask for the smallest radius $r_{ \pm}$that fulfills the condition (22) for all orders $m$, in order to avoid any stray light on the detector. The inner radius of the partial lens for $E_{0}$ will be minimized for:

$$
\mathcal{S}_{m_{ \pm}}\left(\psi_{0}\right)=2 \psi_{0} \rightarrow \psi_{0} \approx 2.62 \text { for }\left[\begin{array}{l}
m_{+}=1 \\
m_{-}=2
\end{array}\right],
$$

and we obtain $r_{\text {min }}\left(E_{0}\right) \approx 5.24 r_{\mathrm{FOV}}$. The condition $\mathcal{S}_{m_{ \pm}}\left(\psi_{0}\right)=2 \psi_{0}$ is equivalent to the relation $\zeta_{ \pm} /\left|1-\zeta_{ \pm}\right|=\psi_{0}$, which represents an equal focal ratio for "blue" $\left(\zeta_{+}>1\right)$ and "red" $\left(\zeta_{-}<1\right)$ detuned X-rays with an energy $E_{2}=$ $2.62 E_{0}$, scattered by the outer ring lens for $E_{1}=E_{0}$. An illustration of this semi-analytical investigation is given in Fig. 10.

The obstruction of the hard band at $E_{2}$ defines the central hole of the aperture and is conservatively set to $1.0 r_{\mathrm{FOV}}$, somewhat more than what the scaling factor $\mathcal{S}_{m}\left(\psi_{0}\right)$ requires. We choose a set of energies between 1 and $20 \mathrm{keV}$. However, X-rays below $5 \mathrm{keV}$ would be mainly absorbed. As a compromise, we take $E_{1}=6.0 \mathrm{keV}$ and $E_{2}=15.7 \mathrm{keV}$ and calculate the lens performance for the case of massive hybrid lens segments (on the left of Fig. 5) in the hard band and coherently stepped versions (Fig. 5, right) for $E_{1}$. The technical data are listed in Table 7. Compared to large diffractive instruments [5], the achromatic gain allows for compact lens radii. In particular, the huge

Table 7 Technical details of the optimized nested two band telescope

\begin{tabular}{lllllllll}
\hline Band & Energy & $w$ & $a$ & $N_{\star}$ & $\mathcal{G}$ & Mat. & $T_{N}$ & $r_{\text {lens }}^{(o)}$ \\
\hline$E_{1}$ & $6.0 \mathrm{keV}$ & 4 & 0.57 & 4480 & 22.2 & $\mathrm{Li}$ & 15 & $1.39 \mathrm{~m}$ \\
$E_{2}$ & $15.7 \mathrm{keV}$ & - & 0.19 & 2750 & 49.5 & $\mathrm{Be}$ & 20 & $0.79 \mathrm{~m}$ \\
\hline
\end{tabular}

The model refers to Fig. 11. See Table 8 for performance data 
Table 8 Performance parameters of the optimized two-band telescope design, according to Fig. 11

\begin{tabular}{llll}
\hline Parameter & Symb. & $E_{1}(6.00 \mathrm{keV})$ & $E_{2}(15.7 \mathrm{keV})$ \\
\hline Resolution & $\Delta \epsilon$ & $0.67 \mathrm{mas}$ & $0.54 \mathrm{mas}$ \\
Spot size & $\varnothing_{\mathrm{PSF}}$ & $0.94 \mathrm{~mm}$ & $0.75 \mathrm{~mm}$ \\
Field of view & $\varnothing_{\mathrm{FOV}}$ & $39 \mathrm{~cm}$ & $30 \mathrm{~cm}$ \\
Focal length & $F$ & $287 \mathrm{~km}$ & \\
$A_{\text {eff }} \times \Delta E$ & & $1212 \mathrm{~cm}^{2} \mathrm{keV}$ & $5331 \mathrm{~cm}^{2} \mathrm{keV}$ \\
\hline
\end{tabular}

geometric area of pure Fresnel objectives is compensated as shown in Table 7, with an almost optimized gain in the hard band.

Apart from the limited FOV, those achromatic dual-band arrangements also compete well with single band telescopes [6] with respect to all other parameters such as resolution, sensitivity and geometrical dimensions. However, the large aspect ratios up to $\sim 2$ must be regarded in particular since properly shaped refractive lens profiles are crucial for an imaging performance free from aberrations. Table 8 overviews the most important variables. Figure 11 sketches the general design of the telescope. The radial dimensions are compared, whereas the focal length is not to scale.

In order to obtain an optimized image quality in $E_{1}$, the comb structure should be detected with a spectral resolution within:

$$
4.4 \times 10^{-4} \leq\left(\frac{\Delta E_{1}}{E_{1}}\right)_{\text {det }} \leq 6.7 \times 10^{-4} .
$$

Albeit currently developed micro calorimeters might match those requirements in the middle-term future for energies around $E_{1} \sim 6 \mathrm{keV}$, their application to hard $\mathrm{X}$-rays beyond $10 \mathrm{keV}$ seems questionable so far.

\subsection{Stray light halos}

The lateral position of a detector spacecraft should be fixed with respect to the optical axis in order to exclude all scattered X-rays. As it follows from

Fig. 11 Schematic view of the achromatic dual band telescope. Both ring lenses are segmented (simplified drawing). The outer halo in the focal plane is only shown in part

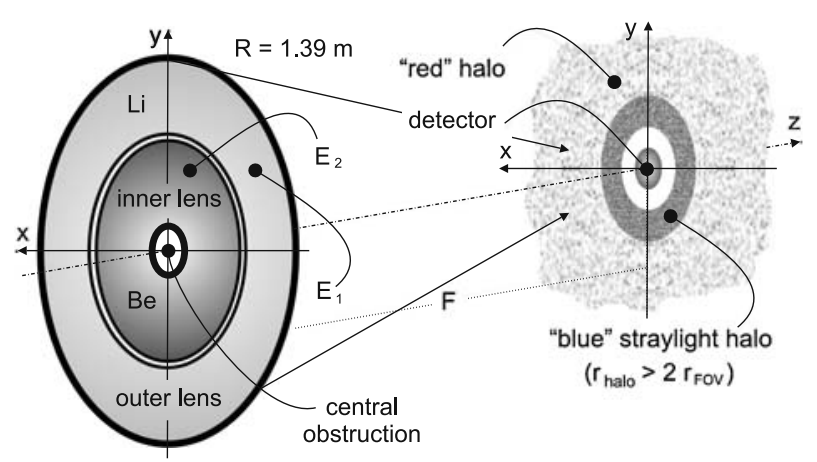



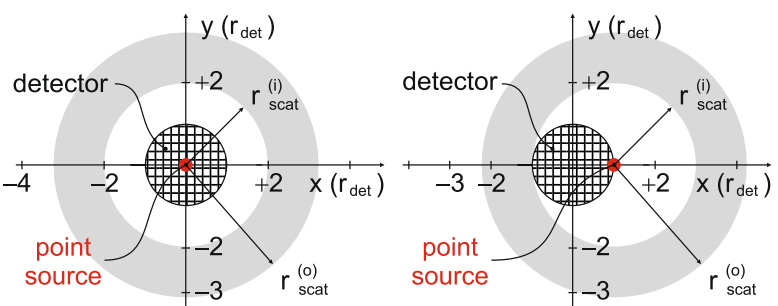

Fig. 12 Secure stray light protection of the detector plate. On the left side, the on-axis point source yields an axially symmetric halo. The halo of extremely displaced point sources on the edge of the detector field just touches but does not contaminate the detector (right picture)

the telescope design based on Fig. 7 and (22), the circular detector field for the energy band $E_{p}$ is protected from any stray light photons in this case: The inner radius of the halo consisting of photons with an energy $E_{p}$ measures at least twice the corresponding detector radius. Point sources which are imaged to an edge pixel of the circular detector field evoke an excentric but still not detected halo (Fig. 12). We estimate the size and intensity of the halos in the focal plane. The inner and outer radii $r_{\text {scat }}^{(i, o)}\left(E_{q}\right)$ of the halo (Fig. 12) are given as:

$$
r_{\text {scat }}^{(i, o)}\left(E_{q}\right)=\left|1-\zeta_{ \pm}^{-1}\right| r_{\text {lens }}^{(i, o)}\left(E_{p}\right)
$$

where $r_{\text {lens }}^{(i, o)}\left(E_{p}\right)$ describes the inner and outer radius of the partial hybrid lens for an energy $E_{p}$. In Fig. 13 the dimensions are illustrated in comparison to the detector plate. However, a misaligned detector spacecraft would also detect X-rays from the surrounding halos. The scattered diffraction efficiencies $P_{\text {scat }}$ result from absorption losses in segments dedicated to $E_{p} \neq E_{q}$ and the focal dispersion. For an equal spectral width as in the regular detection, the luminous power in the halos is found as:

$$
\left(A_{\text {eff }} \times \Delta E\right)\left(E_{q}\right)=\pi \Delta r_{\text {geo }}^{2}\left(E_{p}\right) P_{\text {scat }} \Delta E_{q} .
$$

Fig. 13 Stray light halos for the two-band telescope design according to Fig. 11. The left picture illustrates photons with an energy around $E_{1}$ in the focal plane accidently "imaged" by the inner partial lens for $E_{2}$. On the right, photons with an energy around $E_{2}$, scattered by the outer partial lens for $E_{1}$, surround the detector field

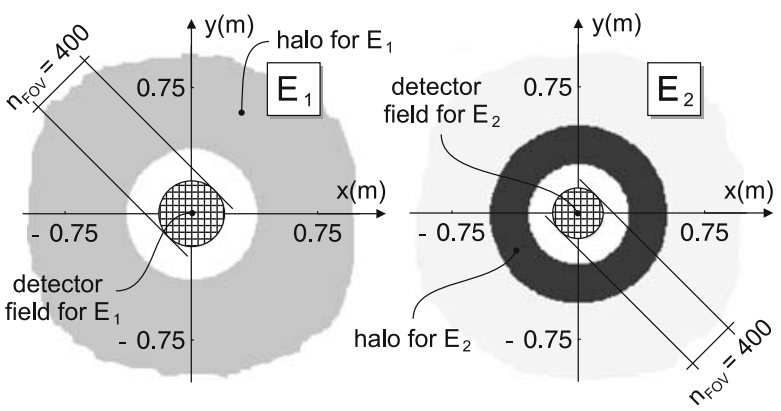


Table 9 Data of the stray light halos from Fig. 13

\begin{tabular}{lllllll}
\hline Band & Energy & $\mathrm{m}$ & $\zeta_{-}$ & $\zeta_{+}$ & $P_{\text {scat }}$ & $\left(A_{\text {eff }} \times \Delta E\right)_{\text {scat }}$ \\
\hline$E_{1}$ & $6 \mathrm{keV}$ & 2 & 0.28 & - & $1.31 \%$ & $1.1 \times 10^{1} \mathrm{~cm}^{2} \mathrm{keV}$ \\
$E_{2}$ & $16 \mathrm{keV}$ & 2,1 & 0.72 & 1.62 & $23.9 \%$ & $5.8 \times 10^{3} \mathrm{~cm}^{2} \mathrm{keV}$ \\
\hline
\end{tabular}

The scattering fraction $P_{\text {scat }}$ refers to the scattered imaging efficiency in the corresponding energy band

The abbreviation $\Delta r_{\text {geo }}^{2}\left(E_{p}\right)$ denotes the squared radial distance $\left[r_{\text {lens }}^{(o)}\left(E_{p}\right)\right]^{2}-$ $\left[r_{\text {lens }}^{(i)}\left(E_{p}\right)\right]^{2}$ for the partial lens dedicated to $E_{p}$. In (32), the scattering efficiency $P_{\text {scat }}$ can be calculated using (7), multiplied with the refractive lens transmission:

$$
P_{\text {scat }}\left(E_{q}\right)=P_{m}\left(E_{q} / E_{p}\right) \times \mathcal{T}_{m}(s) .
$$

In this special case, the "mistuned" $s-$ ratio is given as

$$
s=w_{p}^{-1}\left(N_{\star}\left(E_{p}\right) / N_{0}\left(E_{q}\right)\right) E_{p} E_{q}^{-1} .
$$

The effective "net" energy width $\Delta E_{q}$ is calculated for the regular spectral band around $E_{q}$ :

$$
\Delta E_{1}=\frac{2 E_{1}}{\sqrt{N_{\star}\left(E_{2}\right)}} \quad \text { and } \quad \Delta E_{2}=\frac{E_{2}}{2 \sqrt{N_{\star}\left(E_{1}\right)}} .
$$

Table 9 summarizes the scattering data. Obviously, the soft band lens operates quite efficiently, since only $\sim 1 \%$ of the incident flux is lost in the 2 nd diffraction order. Significant but still acceptable fractions of the hard X-ray component are useless scattered on the other hand by the outer ring lens for $E_{1}$.

\section{Material issues}

Though only very few empirical studies exist on the effect of elementary and other impurities on (X-ray) photons propagating through the lens medium, theoretical predictions clearly describe how photons are scattered by such inhomogeneities [11]. In this work we restrict to predominant absorption losses caused by atomic substitutions in the regular crystal lattice consisting of $\mathrm{Li}$ or Be, respectively.

For $N$ types of atoms with concentrations $c_{n}, 1 \leq n \leq N$ and scattering factors $f_{n}$, the averaged scattering factor $\overline{\mathcal{F}}$ is defined as

$$
\overline{\mathcal{F}} \equiv \sum_{n=1}^{N} c_{n} f_{n} \quad \text { with } \quad \sum_{n=1}^{N} c_{n}=1 .
$$


Since all substitutional atoms have their origin in an imperfect manufacturing process, we can assume randomized positions within the crystal lattice. In agreement with an incoherent superposition of the scattered elementary waves, the impurity atoms would enhance the background in the focal plane over $\operatorname{Re}(\overline{\mathcal{F}})$ and reduce the lens transmission by absorption via $\operatorname{Im}(\overline{\mathcal{F}})$ as well.

We use the data for a commercially available ultra-high purity sample [12] of $\mathrm{Li}$ around $6 \mathrm{keV}$ [13]:

\begin{tabular}{lrr}
$\mathrm{Li}$ & & $\mathrm{f}=3.00226+0.000651 i$ \\
\hline $\mathrm{Na} @ 1.0 \times 10^{-5}: f=11.2067+0.217395 i$ \\
$\mathrm{Ca} @ 5.0 \times 10^{-5}: f=20.1061+2.165730 i$ \\
$\mathrm{Si} @ 5.0 \times 10^{-6}: f=14.3470+0.566843 i$ \\
$\mathrm{Fe} @ 5.0 \times 10^{-6}: f=24.3204+0.642017 i$ \\
$\mathrm{Al} @ 5.0 \times 10^{-6}: f=13.2986+0.420026 i$ \\
$\mathrm{Ni} @ 5.0 \times 10^{-6}: f=26.8777+0.883844 i$
\end{tabular}

In a similar way, the imperfect Beryllium crystal [14] may be analyzed for an energy of $15.7 \mathrm{keV}[13]$ :

\begin{tabular}{lr}
$\mathrm{Be}$ & $: f=4.00065+0.000329 i$ \\
\hline $\mathrm{Cu} @ 5.0 \times 10^{-6}: f=29.2963+1.538240 i$ \\
$\mathrm{~Pb} @ 3.0 \times 10^{-6}: f=75.0876+10.30430 i$ \\
$\mathrm{Cd} @ 2.0 \times 10^{-6}: f=47.3821+1.464520 i$ \\
$\mathrm{Zn} @ 2.5 \times 10^{-5}: f=30.2366+1.746820 i$ \\
$\mathrm{Ni} @ 7.5 \times 10^{-6}: f=28.3358+1.344800 i$ \\
$\mathrm{Co} @ 2.0 \times 10^{-6}: f=27.3538+1.156970 i$ \\
$\mathrm{Mn} @ 2.0 \times 10^{-6}: f=25.3674+0.880966 i$ \\
$\mathrm{Fe} @ 1.7 \times 10^{-5}: f=26.3687+1.019410 i$
\end{tabular}

The averaged scattering factors, which are slightly larger than the pure values, are found as

$$
\begin{aligned}
& \overline{\mathcal{F}}_{\mathrm{Li}}=3.00362+7.74 \times 10^{-4} i, \\
& \overline{\mathcal{F}}_{\mathrm{Be}}=4.00240+4.46 \times 10^{-4} i .
\end{aligned}
$$

The real parts differ by less than $0.1 \%$ from those of pure crystals, whereas deviations beyond $10 \%$ in $\operatorname{Im}(\overline{\mathcal{F}})$ indicate that the contamination with other atoms will predominantly affect the transmission by enhanced absorption rather than the PSF by scattering. Using (36), the parameter $\eta$, an often used measure for the optical quality of the material, can be written as

$$
\eta(E)=\frac{\sum_{j} m_{j} f_{j}^{(2)}(E)}{\sum_{j} m_{j} f_{j}^{(1)}(E)}=\frac{\operatorname{Im}(\overline{\mathcal{F}})}{\operatorname{Re}(\overline{\mathcal{F}})},
$$


where $f_{j}^{(1,2)}$ refer to the energy dependent atomic scattering factor $f_{j}=f_{j}^{(1)}+$ $i f_{j}^{(2)}$. The statistical weight of an atomic type $j$ is denoted by $m_{j}$. We find

$$
\begin{aligned}
& \eta(6.00 \mathrm{keV})=2.58 \times 10^{-4} \quad \text { for } \mathrm{Li} \quad(+18.85 \%), \\
& \eta(15.7 \mathrm{keV})=1.12 \times 10^{-4} \quad \text { for } \mathrm{Be} \quad(+35.61 \%) .
\end{aligned}
$$

Even for contaminations on the ppm level, a significant but still acceptable decline of the optical performance is observed. The relative data, compared to the values of perfect crystals, are listed in $(+\%)$. The usage of ultra-high purity crystals will thus be indispensable for practical implementations of such diffractive-refractive $\mathrm{X}$-ray telescopes in the future.

\section{Simulations}

We demonstrate the astronomical capability of the presented single-focus dual band instrument. As mentioned at the beginning of Section 4, dual band telescopes might be applied to investigations of hardness ratios, probably the simplest form of imaging spectroscopy. It is convenient to assume an ordinary power law whose photon index may vary within $1.2 \lesssim \gamma \lesssim 1.8$.

\subsection{X-ray emission from NGC 4594}

In the following, we consider a typical example of AGN spectra, the "sombrero" galaxy NGC 4594 (http://lheawww.gsfc.nasa.gov/users/.../liner_ llagn.html). As it was measured by ASCA, the spectral slope towards hard $\mathrm{X}$-rays can be described by $\gamma \approx 1.70$ for core emission. Clearly, any synchrotron contributions from potential jets are included in the spectrum shown in Fig. 14. Beyond $10 \mathrm{keV}$, the power law may be extrapolated up to $20 \mathrm{keV}$.

Fig. 14 ASCA X-ray spectrum of the sombrero galaxy NGC 4594. The straight lines describe a power law with the slope $\gamma \approx 1.70$. Figure adopted from the GSFC website (http://lheawww.gsfc.nasa.gov/ users/.../liner_llagn.html)

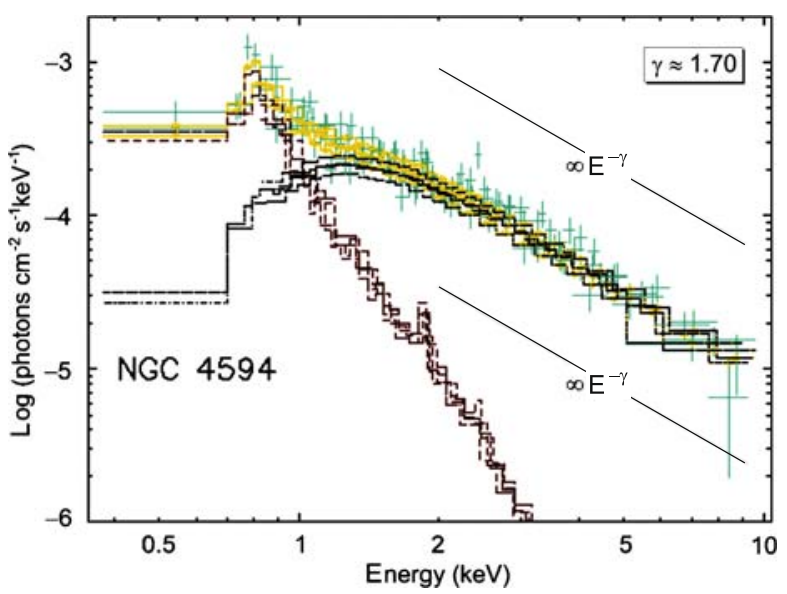

Springer 
The second soft component in Fig. 14 will not be considered within this work. For an energy-dependent luminous power $\left(A_{\text {eff }} \times \Delta E\right)\left(E_{p}\right)$, the partial photon count rate $\Phi\left(E_{p}\right)$ for an energy $E_{p}$ can be written as:

$$
\Phi\left(E_{p}\right) \approx \Phi_{0}\left(\frac{E_{0}}{E_{p}}\right)^{\gamma}\left(A_{\mathrm{eff}} \times \Delta E\right)\left(E_{p}\right),
$$

where $\Phi_{0}$ describes the flux density for the basic energy $E_{0}=6 \mathrm{keV}$ from Fig. 14. Obviously, the count rate in each energy band scales proportional to the effective area and spectral bandwidth of the corresponding ring lens for $E_{1}$ and $E_{2}$, respectively. These luminous power data $\left(A_{\text {eff }} \times \Delta E\right)\left(E_{p}\right)$ are given in Table 8.

The flux density at $15.7 \mathrm{keV}$ amounts to about $20 \%$ of the corresponding one at $6 \mathrm{keV}$. Since the telescope provides an almost inverse sensitivity, we expect similar count rates in both energy bands. Integrated to an exposure time $\Delta t_{\mathrm{obs}}$ of $10^{6} \mathrm{~s}$, photon numbers greater than $2 \times 10^{4}$ in the soft and hard band may be expected. In general, the target photons will be distributed among the number of pixels corresponding to the resolution of the X-ray source. However, in the case of NGC 4594, the central black hole has been estimated to $10^{9} M_{\odot}$ [15]. The corresponding gravitational radius $R_{S}$ of $2.9 \times 10^{12} \mathrm{~m}$ implies an overall diameter of the accretion disk of at least $10^{15}-10^{16} \mathrm{~m}$, depending on the theoretical model. The angular resolution of $\lesssim 10^{-3}$ arcsec yields an estimated length scale of the same order, about $10^{15} \mathrm{~m}$. Though the detailed structure of the accretion disk will hardly be resolved by our telescope, an optimal signalto-noise ratio (SNR) may be expected for signal photons distributed over a few pixels. In particular, an assumed accretion disk which is extended to $10^{3} R_{s}$ would correspond to an angular diameter of 4.2 mas.

Radio observations [15] with an angular resolution of $5 \times 10^{-3}$ arcsec unearthed no extended X-ray emission aside from the central nucleus. Other investigations speculated on the existence of some directed outflows, e.g. jets [16]. If present on length scales in the order of $10^{-3}$ arcsec, they could be resolved to several pixels. Assuming dimensions of $A_{\text {pix }}=(395 \times 395) \mu \mathrm{m}^{2}$, we expect an image similar to that presented in Fig. 15.

Fig. 15 Central region of NGC 4594, as it might be seen by the two-band telescope. The soft and hard bands are shown on the left and right, respectively

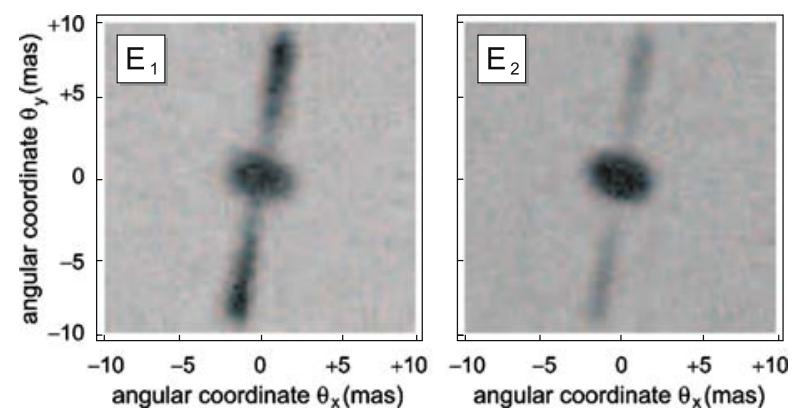

angular coordinate $\theta_{\mathrm{x}}$ (mas) 
8.2 Signal-to-noise ratio

We estimate the SNR in a pixel numbered by $(i, j)$ :

$$
\operatorname{SNR}_{i j}\left(E_{p}\right)=\frac{S_{i j}\left(E_{p}\right)}{\sqrt{S_{i j}\left(E_{p}\right)+B_{i j}\left(E_{p}\right)}}
$$

for signal counts $S_{i j}$ and background events $B_{i j}$. The total number of signal counts, integrated from (40) for an observation time of $10^{6} \mathrm{~s}$, amounts to $2.87 \times 10^{4}$ and $2.46 \times 10^{4}$, respectively, summed up from both disk and jet contributions. We presume a hardness ratio of 2.5 for disk-to-jet conversion between the soft and the hard band emission. Thus we obtain the total number of signal counts in the disk $\left(S_{\text {disk }}\right)$ and jets $\left(S_{\text {jets }}\right)$, respectively (Table 10$)$. In order to estimate the diffuse XRB, an overall power law of the form $n_{b}(E)=n_{0} E^{-\Gamma}$, with $n_{0}=(9.8 \pm 0.3) \mathrm{s}^{-1} \mathrm{~cm}^{-2} \mathrm{keV}^{-1} \mathrm{sr}^{-1}$ and $\Gamma=1.42 \pm 0.02$ was taken from Revnivtsev et al. [17]. The number of background counts is roughly given as

$$
n_{b}\left(E_{p}\right)=\frac{1}{5} \pi n_{0} E_{p}^{-\Gamma} \Delta t_{\mathrm{obs}} A_{\text {pix }} \Delta E_{p},
$$

if the radiation is collimated in front of the detector to a solid angle of $10 \%$ of $2 \pi$. Following (17) and Table 7, the net achromatic bandwidth $\Delta E_{p}$ amounts to $90 \mathrm{eV}$ at $E_{1}$ and $600 \mathrm{eV}$ around $E_{2}$. We find about 68 natural background counts per pixel within the assumed exposure time of $10^{6} \mathrm{~s}$ for the soft band and 115 disturbing photons around $15.7 \mathrm{keV}$.

The SNR may be thus as good as 8.3 for $6 \mathrm{keV}$ and 10 in the hard band, as it is confirmed by numerical simulations: For each pixel within the FOV, the background level is superimposed by the presumed original intensity distribution of the target. The result is convoluted with the discrete PSF and "randomized" by an artificial Poisson noise (see Fig. 15).

Obviously, almost nothing is known about the actual length and brightness of the synchrotron jets and the accretion disk might be 10 times smaller than assumed above. However, our simulations prove the feasibility of fruitful observations using two-band achromatic telescopes.

Table 10 Photon counts and covered number of pixels \#(pix) for the modeled disk-jet system in the core of NGC 4594

\begin{tabular}{lll}
\hline Target region & $E_{1}(6.0 \mathrm{keV})$ & $E_{2}(15.7 \mathrm{keV})$ \\
\hline$S_{\text {disk }} / \#$ (pix) & $8.32 \times 10^{3} / 105$ & $1.75 \times 10^{4} / 105$ \\
$S_{\text {jets }} / \#$ (pix) & $2.04 \times 10^{4} / 185$ & $7.13 \times 10^{3} / 185$ \\
\hline
\end{tabular}

Following the data from Table 8 , each pixel covers $(0.28 \times 0.28) \mathrm{mas}^{2}$ in the focal plane 


\section{Conclusion}

An extension to single-band hybrid Fresnel X-ray telescopes for spectroscopic applications is developed. The detectable energy range may be doubled at least, using several partial instruments with a common focal length. An efficient design relies on segmented, nested ring lenses for the simultaneous imaging of two energy bands. The "achromatic gain" is introduced as an essential concept for optimized assemblies of that type. Central detector units minimize the natural as well as artificial background noise. The observational sensitivities of the soft and hard band are very suitable for measurements of hardness ratios for power-law emitting sources like AGN or X-ray binaries.

Further theoretical steps should enhance the luminous power and the number of energy bands. From a technological point of view, dedicated detectors for the spectral structure of stepped hybrid profiles are of great interest. Other challenges point to investigations of low-Z lens materials and an accurate control system for the positioning of lens and detector in space-problems that will be attended to forthcoming publications.

Acknowledgements This work was financially supported in part by the Heidenhain foundation, Traunreut (Germany). The authors gratefully recognize encouraging discussions with Joachim Trümper. Special thanks are dedicated to Karsten Jessen who carefully read the manuscript.

Open Access This article is distributed under the terms of the Creative Commons Attribution Noncommercial License which permits any noncommercial use, distribution, and reproduction in any medium, provided the original author(s) and source are credited.

\section{References}

1. Skinner, G.K., et al.: Fresnel lenses for X-ray and Gamma-ray astronomy. Proc. SPIE 5168, 459 (2004)

2. Skinner, G.K., et al.: Diffractive-refractive optics for high energy astronomy-II. Variations on the theme. Astron. Astrophys. 383, 352 (2002)

3. Gorenstein, P.: Role of diffractive and refractive optics in X-ray astronomy. Proc. SPIE 5168, 411 (2004)

4. Skinner, G.K.: Design and imaging performance of achromatic diffractive-refractive X-ray and gamma-ray Fresnel lenses. Appl. Opt. 43, 4845 (2004)

5. Braig, C., Predehl, P.: Large-scale diffractive X-ray telescopes. Exp. Astron. 21, 101 (2006)

6. Braig, C., Predehl, P.: Efficient Fresnel X-ray optics made simple. Appl. Opt. 46, 2586 (2007)

7. Michette, A.G.: Optical Systems for Soft X-rays. Plenum, New York (1986)

8. Kirz, J.: Phase zone plates for X-rays and the extreme UV. JOSA 64(3), 301 (1974)

9. Yang, B.X.: Fresnel and refractive lenses for X-rays. Nucl. Instrum. Methods A 328, 578 (1993)

10. Wang, Y., et al.: Achromatic Fresnel optics for wideband extreme-UV and X-ray imaging. Nature 424, 50 (2003)

11. Guinier, A.: X-ray Diffraction in Crystals, Imperfect Crystals and Amorphous Bodies. Dover, New York (1994)

12. American Elements: American Elements homepage. Los Angeles, Calif. http://www. americanelements.com (2006)

13. Henke, B.L., et al.: X-ray interactions: photoabsorption, scattering, transmission, and reflection at $\mathrm{E}=50-30000 \mathrm{eV}, \mathrm{Z}=1-92$. At. Data Nucl. Data Tables 54, 181 (1993) 
14. Goode, G.C., et al.: The determination of some impurities in high purity Beryllium by differential cathode-ray polarography. Analyst 91, 719 (1966)

15. Pellegrini, S., et al.: An XMM-Newton and Chandra investigation of the nuclear accretion in the sombrero galaxy (NGC 4594). Astrophys. J. 597, 175 (2003)

16. Di Matteo, T., et al.: Limits on the accretion rates onto massive black holes in nearby galaxies. Astrophys. J. 547, 731 (2001)

17. Revnivtsev, M., Gilfanov, M., Sunyaev, R., Jahoda, K., Markwardt, C.: The spectrum of the cosmic X-ray background observed by RXTE/PCA. Astron. Astrophys. 411, 329-334 (2003) 